\title{
A practical approach to estimating optic disc dose and macula dose without treatment planning in ocular brachytherapy using ${ }^{125}$ I COMS plaques
}

Yongsook C. Lee, Shih-Chi Lin and Yongbok Kim ${ }^{*}$

\begin{abstract}
Background: It has been reported that proximity of the tumor to the optic disc and macula, and radiation dose to the critical structures are substantial risk factors for vision loss following plaque brachytherapy. However, there is little dosimetry data published on this. In this study, therefore, the relationship between distance from tumor margin and radiation dose to the optic disc and macula in ocular brachytherapy using ${ }^{125}$ I Collaborative Ocular Melanoma Study (COMS) plaques was comprehensively investigated. From the information, this study aimed to allow for estimation of optic disc dose and macula dose without treatment planning.

Methods: An in-house brachytherapy dose calculation program utilizing the American Association of Physicists in Medicine Task Group-43 U1 formalism with a line source approximation in a homogenous water phantom was developed and validated against three commercial treatment planning systems (TPS). Then optic disc dose and macula dose were calculated as a function of distance from tumor margin for various tumor basal dimensions for seven COMS plaques (from $10 \mathrm{~mm}$ to $22 \mathrm{~mm}$ in $2 \mathrm{~mm}$ increments) loaded with commercially available ${ }^{125}$ s seeds models (IAl-125A, 2301 and 125.S16). A prescribed dose of 85 Gy for an irradiation time of $168 \mathrm{~h}$ was normalized to a central-axis depth of $5 \mathrm{~mm}$. Dose conversion factors for each seed model were obtained by taking ratios of total reference air kerma per seed at various prescription depths (from $1 \mathrm{~mm}$ to $10 \mathrm{~mm}$ in $1 \mathrm{~mm}$ intervals) to that at $5 \mathrm{~mm}$.

Results: The in-house program demonstrated relatively similar accuracy to commercial TPS. Optic disc dose and macula dose decreased as distance from tumor margin and tumor basal dimension increased. Dose conversion factors increased with increasing prescription depth. There existed dose variations $(<8 \%)$ among three ${ }^{125}$ I seed models. Optic disc dose and macula dose for each COMS plaque and for each seed model are presented in a figure format. Dose conversion factors for each seed model are presented in a tabular format.
\end{abstract}

Conclusions: The data provided in this study would enable clinicians in any clinic using ${ }^{125}$ I COMS plaques to estimate optic disc dose and macula dose without dose calculations.

Keywords: Ocular brachytherapy, ${ }^{125}$, , COMS plaques, Optic disc dose, Macula dose

\footnotetext{
* Correspondence: yongbokkim@email.arizona.edu

Department of Radiation Oncology, The University of Arizona, 3838 N.

Campbell Avenue, Building \#2, Tucson, AZ 85719, USA
}

(c) The Author(s). 2018 Open Access This article is distributed under the terms of the Creative Commons Attribution 4.0 International License (http://creativecommons.org/licenses/by/4.0/), which permits unrestricted use, distribution, and reproduction in any medium, provided you give appropriate credit to the original author(s) and the source, provide a link to the Creative Commons license, and indicate if changes were made. The Creative Commons Public Domain Dedication waiver (http://creativecommons.org/publicdomain/zero/1.0/) applies to the data made available in this article, unless otherwise stated. 


\section{Background}

Plaque brachytherapy is currently the most common treatment option for early stage or medium-sized intraocular tumors ( $\leq 10 \mathrm{~mm}$ in apical height and $\leq 16 \mathrm{~mm}$ in diameter for uveal melanomas) [1-3]. It offers equivalent tumor control and better quality of life such as eye preservation and vision retention in comparison to enucleation [3-5]. Various plaque designs were proposed and are clinically used in major institutions [6-8]. Nonetheless, Collaborative Ocular Melanoma Study (COMS) plaques have been widely used in most clinics since the COMS established standardized methods of plaque brachytherapy for medium-sized choroidal melanomas [3].

In plaque brachytherapy for intraocular tumors, major critical structures related to vision are lens, optic nerve (optic disc) and macula (fovea). A cataract, clouding of the lens, is the most common radiotherapy contraindication but a surgery can restore vision loss due to cataracts. On the other hand, radiation damage to the optic disc and macula can cause permanent vision loss which is usually not recoverable. Several studies reported outcomes for vision deterioration/loss following plaque brachytherapy $[4,5,9-13]$. Some of the studies revealed that proximity of the tumor to the optic disc and fovea, and radiation dose are substantial risk factors for vision loss $[4,5,9]$. However, there is a paucity of literature on the relationship between proximity of the tumor to the vision-related critical structures and radiation dose to them in plaque brachytherapy.

Therefore, this study has comprehensively examined the relationship between distance from tumor margin and radiation dose to the optic disc or macula in ocular brachytherapy using ${ }^{125}$ I COMS plaques through a dosimetry study. By providing the dosimetry data, this study aims to enable clinicians (both ophthalmologist and radiation oncologist) in any clinic or institution using ${ }^{125} \mathrm{I}$ COMS plaques to predict optic disc dose and macula dose at the time of tumor size measurements without dose calculations in a treatment planning system (TPS).

The American Association of Physicists in Medicine (AAPM) Task Group (TG) 129 recommends that in dose calculations, heterogeneity corrections be accounted for non-tissue materials such as gold-alloy backing and silastic seed carrier insert in the plaque [3]. As of today, however, there is no commercially available TPS taking into account heterogeneity corrections. Furthermore, the hybrid method, homogeneous dose calculations multiplied by known heterogeneity correction factors, suggested by the AAPM TG 129, is limited to the obsolete ${ }^{125}$ I seed model 6711 [3] and there is no correction factor provided for currently available ${ }^{125} \mathrm{I}$ seed models. Herein, in current clinical practice, the AAPM TG-43 dosimetry formalism with a line source approximation in a homogeneous water medium is widely used. In this study, dose calculations were performed based on the current clinical practice.

\section{Methods}

Determination of parameters required for treatment planning Following COMS protocols [14], five parameters required for treatment planning were determined in an ophthalmologist's office. Tumor basal dimension at center in the direction from optic disc (BD, parameter \#1) and distance from optic disc to tumor margin (DT, parameter \#2) were measured in a fundus diagram. Tumor basal dimension at center in the direction from macula (BM, parameter \#3) and distance from macula to tumor margin (MT, parameter \#4) were also measured in the same fundus diagram. Tumor height (parameter \#5), which determines a prescription depth, was measured using ultrasound. The fundus diagram in Fig. 1a illustrates $\mathrm{BD}, \mathrm{DT}, \mathrm{BM}$ and $\mathrm{MT}$ of the tumor and the cross section diagram of the eye in Fig. 1b shows apical height of the tumor. Adequate plaque size was determined by
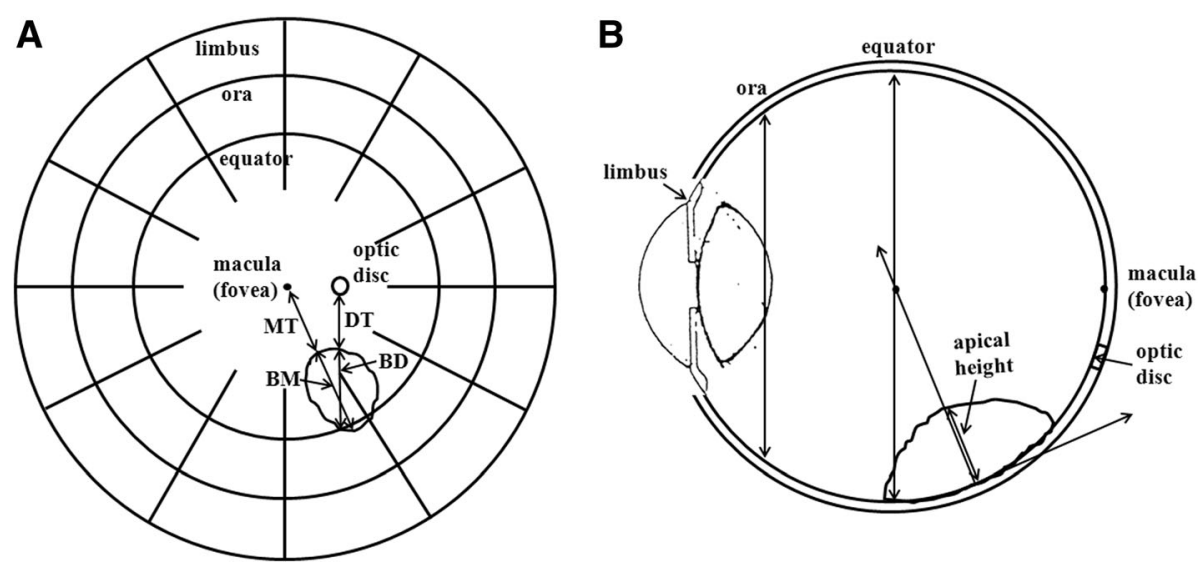

Fig. 1 a The fundus diagram illustrating BD, DT, BM and MT of the tumor and $\mathbf{b}$ the cross section diagram of the eye showing apical height of the tumor 
adding a margin of $2-3 \mathrm{~mm}$ to the largest tumor dimension. Then this information was sent to the department of radiation oncology for treatment planning.

\section{Validation of our in-house brachytherapy dose calculation program}

For efficient calculations of optic disc dose and macula dose as a function of parameters mentioned above, an in-house brachytherapy dose calculation program was developed in MATLAB $^{\circ}$ software (vR2016a, MathWorks, Natick, MA) and validated against three commercial TPS for benchmark calculations in the literature [15]. The conventional AAPM TG-43 Update (TG-43 U1) dosimetry formalism with a line source approximation in a homogeneous water medium was incorporated into the in-house program. Parameters for the TG-43 U1 formalism including radial dose function (Table II in TG-43 U1) and anisotropy function (Table $\mathrm{V}$ in TG-43 U1) were taken from the TG-43 U1 [16]. The step size over distance " $r$ " and polar angle " $\theta$ " for radial dose function and anisotropy function was coarser in the TG-43 U1 than that in Rivard et al. study. In our in-house program, linear interpolation was used to obtain radial dose function and anisotropy function values, while in Rivard et al., log-linear interpolation was used for radial dose function values and linear interpolation for anisotropy function values. Seed coordinates for COMS plaques taken from Table I in the AAPM TG 129 [3] were also incorporated into the program. In Rivard et al.'s benchmark test, doses at several points along the central-axis and at off-axis points for organs at risk (OARs) were calculated in commercial TPS for a $16 \mathrm{~mm}$ COMS plaque loaded with ${ }^{125}$ I seeds (Amersham Oncoseed 6711). The TPS include $\mathrm{P}^{3}$ (Pinnacle ${ }^{3}$, v8.0dpl, Philips Medical Systems, Cleveland, OH), BV (BrachyVision ${ }^{\mathrm{Tm}}$, v8.1, Varian Medical Systems, Inc., Palo Alto, CA) and PS (Plaque Simulator, v5.3.9, Eye Physics LLC, Los Alamitos, CA) which all use a line source approximation in homogeneous water phantoms [15]. In all three TPS, air-kerma strength $\left(S_{k}\right)$ per seed (unit: $U=\mu G^{2}{ }^{2} h^{-1}$ ) was kept the same (4.572 $\mathrm{U})$ to deliver approximately $85 \mathrm{~Gy}$ to a central-axis depth of $5 \mathrm{~mm}$ for an irradiation time of $100 \mathrm{~h}$. The central-axis depth is the distance along the plaque central-axis from the inner sclera. Central-axis and off-axis point doses for this benchmark test were calculated in our in-house program. Total reference air kerma (TRAK $=S_{k} \times$ irradiation time) per seed (unit: $\left.\mu \mathrm{Gym}^{2}\right)$ was kept the same $(4.572 \mathrm{U} \times 100 \mathrm{~h})$ as in the benchmark test. Then, for each point, our data were compared with those in Rivard et al.'s study by computing the modulus of a relative percent difference in dose using the following equation:

$$
\left|D_{\text {diff }}^{\text {Rel }}(\%)\right|=\frac{\left|D_{\text {in }}-D_{\text {ref }}\right|}{D_{\text {ref }}} \times 100
$$

where $D_{\text {in }}$ is dose calculated in our in-house program and $D_{\text {ref }}$ is reference dose from Rivard et al.'s study.

\section{Calculations of optic disc dose and macula dose for standard COMS plaques loaded with ${ }^{125}$ I seeds}

Optic disc dose and macula dose for standard COMS plaques were calculated in the in-house program. Dose calculations were performed as a function of distance from tumor margin (DT or MT) up to $10 \mathrm{~mm}$ for various tumor basal dimensions (BD or BM) $(<20 \mathrm{~mm}$ in $2 \mathrm{~mm}$ intervals). A prescribed dose of $85 \mathrm{~Gy}$ for an irradiation time of $168 \mathrm{~h}$ was normalized at a central-axis depth of $5 \mathrm{~mm}$. The calculations were performed for all seven different-sized COMS plaques (from $10 \mathrm{~mm}$ to $22 \mathrm{~mm}$ in diameter in $2 \mathrm{~mm}$ increments) and for three currently, commercially available ${ }^{125}$ I seeds models (IsoAid Advantage IAI-125A, Best Industries 2301 and Bebig I25.S16) of the seed models listed in the AAPM TG 129 [3]. Parameters in the dosimetry formalism for the three seed models were taken from the AAPM TG-43 U1 [16] and supplement to the AAPM TG-43 U1 [17].

\section{Generation of dose conversion factors for different prescription depths}

Since a prescription depth is determined based on the tumor apex (COMS protocols [14] or American Brachytherapy Society (ABS) guidelines [18]) and it is not always $5 \mathrm{~mm}$, dose conversion factors for different prescription depths were generated in the in-house program. Optic disc dose and macula dose were calculated for prescription depths from $1 \mathrm{~mm}$ to $10 \mathrm{~mm}$ in $1 \mathrm{~mm}$ intervals. The prescribed dose (85 Gy) and irradiation time $(168 \mathrm{~h})$ were kept the same as for prescription depth of $5 \mathrm{~mm}$. Then ratios of TRAK per seed to obtain 85 Gy to each prescription depth to that to $5 \mathrm{~mm}$ were taken as dose conversion factors. The calculations were performed for all seven COMS plaques and for the three seed models mentioned above.

\section{Results \\ Validation of our in-house brachytherapy dose calculation program}

Table 1 presents the comparison of central-axis dose values for a $16 \mathrm{~mm}$ COMS plaque loaded with ${ }^{125} \mathrm{I}$ seeds (model 6711) between our in-house program and three TPS used in Rivard et al.'s study [15]. Max| $\mathrm{D}_{\text {diff }}^{\mathrm{Rel}}(\%) \mid$ is the largest modulus of relative percent differences in dose between the two studies. Max $\mid D_{\text {diff }}^{\text {Rel }}$ (\%)| ranged from $0.32 \%$ to $2.35 \%$ and the largest 
Table 1 The comparison of central-axis dose values (in Gy) for a $16 \mathrm{~mm}$ COMS plaque loaded with ${ }^{125}$ I seeds (model 6711) calculated in our in-house program with those in three commercial treatment planning systems (TPS) in Rivard et al.'s study

\begin{tabular}{|c|c|c|c|c|c|c|}
\hline \multirow{2}{*}{$\begin{array}{l}\mathrm{d} \\
(\mathrm{mm})\end{array}$} & \multirow[t]{2}{*}{ CAX points } & \multirow{2}{*}{$\begin{array}{l}\text { Current } \\
\text { Study }\end{array}$} & \multicolumn{3}{|c|}{ Data from Table II in Rivard et al. [15] } & \multirow[t]{2}{*}{$\operatorname{Max}\left|D_{\text {diff }}^{R e l}(\%)\right|$} \\
\hline & & & $\overline{p^{3}}$ & BV & PS & \\
\hline-1.0 & Outer sclera & 336.18 & 341 & 340 & 339 & 1.41 \\
\hline 0.0 & Inner sclera & 258.79 & 261 & 261 & 260 & 0.85 \\
\hline 1.0 & & 202.10 & 203 & 203 & 202 & 0.44 \\
\hline 2.0 & & 160.48 & 161 & 161 & 160 & 0.32 \\
\hline 3.0 & & 128.68 & 129 & 129 & 128 & 0.53 \\
\hline 4.0 & & 103.91 & 104 & 104 & 103 & 0.88 \\
\hline 5.0 & Rx depth & 84.52 & 84.4 & 84.5 & 83.9 & 0.74 \\
\hline 6.0 & & 69.26 & 69.2 & 69.2 & 68.8 & 0.67 \\
\hline 7.0 & & 57.27 & 57.2 & 57.2 & 56.9 & 0.65 \\
\hline 8.0 & & 47.73 & 47.7 & 47.7 & 47.4 & 0.70 \\
\hline 9.0 & & 40.12 & 40.0 & 40.0 & 39.8 & 0.81 \\
\hline 10.0 & & 34.00 & 33.9 & 33.9 & 33.7 & 0.89 \\
\hline 11.3 & Eye center & 27.73 & 27.6 & 27.6 & 27.5 & 0.83 \\
\hline 15.0 & & 16.45 & 16.3 & 16.3 & 16.3 & 0.95 \\
\hline 20.0 & & 9.02 & 8.87 & 8.89 & 8.84 & 2.04 \\
\hline 22.6 & Opposite retina & 6.83 & 6.70 & 6.70 & 6.67 & 2.35 \\
\hline
\end{tabular}

$\mathrm{P}^{3}$, BV and PS represent Pinnacle, BrachyVision and Plaque Simulator, respectively. Dose values were calculated for a prescribed dose of approximately 85 Gy to a central-axis depth of $5 \mathrm{~mm}\left(\mathrm{~S}_{\mathrm{k}}=4.752 \mathrm{U}\right.$ and irradiation time $\left.=100 \mathrm{~h}\right)$ using a line source approximation of the AAPM TG-43 formalism and homogeneous water phantoms. $\operatorname{Max}\left|D_{\text {diff }}^{\text {Rel }}(\%)\right|$ is the largest modulus of relative percent differences in dose between the two studies

difference $(2.35 \%)$ occurred at the farthest dose point (22.6 mm, opposite retina).

Table 2 presents the comparison of doses at OAR points (fovea, optic disc center, lens center and lacrimal glad center) for four different plaque positions (\#1-\#4) between our study and Rivard et al.'s. From Fig. 3 in Rivard et al., the plaque positions \#1, \#2, \#3 and \#4 were centered on equator on temporal side ( 9 o'clock), on nasal side (3 o'clock), on superior side (12 o'clock) and on inferior side (6 o'clock), respectively [15]. Coordinates for the OARs were taken from Rivard et al. [15]. $\mid D_{\text {diff }}^{\text {Rel }}(\%$ )| was defined in the same way as for the central axis dose comparison except that $D_{\text {ref }}$ was an average value of off-axis doses calculated from all TPS [15]. Off-axis dose differences between the two studies ranged from $0.40 \%$ to $1.52 \%$ except for the lacrimal gland point for plaque positions \#1 (4.39\%) and \#3 (4.40\%).

\section{Optic disc dose and macula dose for standard COMS} plaques loaded with ${ }^{125}$ I seeds

Optic disc dose and macula dose for standard COMS plaques loaded with ${ }^{125}$ I seeds (model IAI-125A) are shown in Figs. 2 and 3, respectively. Figure 2a-g presents optic disc dose as a function of DT up to $10 \mathrm{~mm}$ for various BDs in $2 \mathrm{~mm}$ intervals for seven COMS plaques when 85 Gy is prescribed at a central-axis depth of $5 \mathrm{~mm}$ for an irradiation time of $168 \mathrm{~h}$. Optic disc dose decreases with increasing DT and increasing BD. For the plaques $\geq 16 \mathrm{~mm}$, however, there exist some regions where optic disc dose does not change much with DT (i.e., flat regions in Fig. $2 \mathrm{~d}-\mathrm{g}$ ). This usually occurs within short distances $(\leq 5 \mathrm{~mm}$ of $\mathrm{DT})$ when BD is less than or equal to $5 \mathrm{~mm}$. For example, for the $16 \mathrm{~mm}$ COMS plaque (Fig. $2 \mathrm{~d}$ ), the graph for $\mathrm{BD}=1 \mathrm{~mm}$ does not vary a lot at $<3 \mathrm{~mm}$ of DT and the graph for $\mathrm{BD}=3 \mathrm{~mm}$ does not change much at $<2 \mathrm{~mm}$ of DT, respectively. Plaque size also determines optic disc dose and the shape of dose curves. Macula dose is displayed in Fig. 3a-g as a function of MT for various BMs. Similar patterns to optic disc dose are observed. Optic disc dose and macula dose for the other two seed models (2301 and I25.S16) are presented as Additional file 1 (data not shown here).

There are variations of optic disc dose and macula dose among seed models. The maximum relative differences (\%) in optic disc dose between IAI-125A and 2301, between IAI-125A and I25.S16, and between 2301 and I25.S16 are 7.74, 5.89 and 5.28\%, respectively. Corresponding maximum relative differences (\%) for macula dose are $7.39,5.64$ and $5.28 \%$.

Dose conversion factors for different prescription depths Dose conversion factors for different prescription depths from $1 \mathrm{~mm}$ to $10 \mathrm{~mm}$ in $1 \mathrm{~mm}$ intervals for standard COMS plaques loaded with ${ }^{125}$ I seed (model IAI-125A) are tabulated in Table 3. Based on the COMS protocols 
Table 2 The comparison of dose values (in Gy) at organs at risk points (fovea, optic disc center, lens center and lacrimal glad center) for four different plaque positions (\#1-\#4) [15] of the $16 \mathrm{~mm}$ COMS plaque loaded with ${ }^{125}$ seeds (model 6711) calculated in our in-house program with average off-axis dose values calculated from treatment planning systems used in Rivard et al.'s study

\begin{tabular}{|c|c|c|c|c|}
\hline Plaque position & Off-axis location & Current Study & Data from Table III in Rivard et al. [15] & $\left|D_{\text {diff }}^{R e l}(\%)\right|$ \\
\hline \multirow[t]{4}{*}{$\# 1$} & Fovea & 16.50 & 16.3 & 1.25 \\
\hline & Optic disc & 11.29 & 11.2 & 0.79 \\
\hline & Lens & 21.59 & 21.5 & 0.40 \\
\hline & Lacrimal gland & 40.92 & 39.2 & 4.39 \\
\hline \multirow[t]{4}{*}{ \#2 } & Fovea & 16.50 & 16.3 & 1.25 \\
\hline & Optic disc & 28.02 & 27.6 & 1.52 \\
\hline & Lens & 21.59 & 21.5 & 0.40 \\
\hline & Lacrimal gland & 7.19 & 7.1 & 1.22 \\
\hline \multirow[t]{4}{*}{ \#3 } & Fovea & 16.50 & 16.3 & 1.25 \\
\hline & Optic disc & 16.48 & 16.3 & 1.08 \\
\hline & Lens & 21.59 & 21.5 & 0.40 \\
\hline & Lacrimal gland & 40.92 & 39.2 & 4.40 \\
\hline \multirow[t]{4}{*}{$\# 4$} & Fovea & 16.50 & 16.3 & 1.25 \\
\hline & Optic disc & 16.48 & 16.3 & 1.08 \\
\hline & Lens & 21.59 & 21.5 & 0.40 \\
\hline & Lacrimal gland & 7.19 & 7.1 & 1.22 \\
\hline
\end{tabular}

Dose values were calculated for a prescribed dose of approximately $85 \mathrm{~Gy}$ to a central-axis depth of $5 \mathrm{~mm}\left(\mathrm{~S}_{\mathrm{k}}=4.752 \mathrm{U}\right.$ and irradiation time $\left.=100 \mathrm{~h}\right)$ using a line source approximation of the AAPM TG-43 formalism and homogeneous water phantoms. $\left|\mathrm{D}_{\text {diff }}^{\text {Rel }}(\%)\right|$ is a modulus of a relative percent difference in dose between the two studies

for tumors with apical height $<5 \mathrm{~mm}$ [14], dose conversion factors were normalized to a depth of $5 \mathrm{~mm}$. The factors increase with increasing prescription depth. The factors increase with increasing plaque size for a prescription depth $<5 \mathrm{~mm}$ but the opposite is observed for a depth $>5 \mathrm{~mm}$. Table 3 is used for both optic disc dose and macula dose estimation when a prescription depth is not $5 \mathrm{~mm}$. Dose conversion factors for the other two ${ }^{125}$ I seed models (2301 and I25.S16) are provided as Additional file 2 (data not shown here). The differences of the factors among seed models increase with increasing prescription depth and decreasing plaque size. Maximum absolute differences between IAI-125A and 2301, between IAI-125A and I25.S16, and between 2301 and I25.S16 are 0.08, 0.1 and 0.02 , respectively.

\section{Estimation of optic disc dose without dose calculations: clinical application of this study}

Optic disc dose (Fig. 2), macula dose (Fig. 3) and dose conversion factors (Table 3) presented in the current study can be conveniently used in clinic. As an example, there is a clinical case in which BD is $3 \mathrm{~mm}$, DT is $3 \mathrm{~mm}$ and apical height is $4 \mathrm{~mm}$. A clinician wants to prescribe 85 Gy to the tumor apex (i.e., $4 \mathrm{~mm}$ ) using a $10 \mathrm{~mm}$ COMS plaque loaded with ${ }^{125} \mathrm{I}$ seeds (model IAI-125A). From the data obtained in this study, optic disc dose is about 145 Gy for the $10 \mathrm{~mm}$ COMS plaque and for a prescription depth of $5 \mathrm{~mm}$ (Fig. 2a). The dose conversion factor for the $10 \mathrm{~mm}$ COMS plaque and for a prescription depth of $4 \mathrm{~mm}$ is 0.77 (Table 3). Thus, for this clinical case, expected optic disc dose is 112 Gy $(=145 \mathrm{~Gy} \times 0.77)$ which is lower than that when prescribed at $5 \mathrm{~mm}$ by $33 \mathrm{~Gy}$. If tumor apex is higher (for instance, $6 \mathrm{~mm}$ ) for the same tumor, optic disc dose for the same ${ }^{125}$ I seed model is about 184 Gy $(=145 \mathrm{~Gy} \times$ 1.27) (Table 3).

\section{Discussion}

Our in-house brachytherapy dose calculation program demonstrated similar accuracy in brachytherapy dose calculations to commercial TPS. As presented in Tables 1 and 2, dose differences at central-axis and off-axis points between our in-house program and the three TPS used in Rivard et al.'s study were $<2.4 \%$ except for the lacrimal gland point for plaque positions $\# 1$ and $\# 3$. Some seeds in plaque positions \#1 and \#3 have small polar angles $(<40$ degrees $)$ to the lacrimal gland point. At small polar angles, anisotropy function values vary more dramatically with polar angle than at large polar angles, leading to larger uncertainty in the interpolation of anisotropy function values. As mentioned in the Methods, Rivard et al.'s study used smaller step size over distance " $r$ " and polar angle " $\theta$ " for anisotropy function than our in-house program (AAPM TG-43 U1), causing larger dose differences 

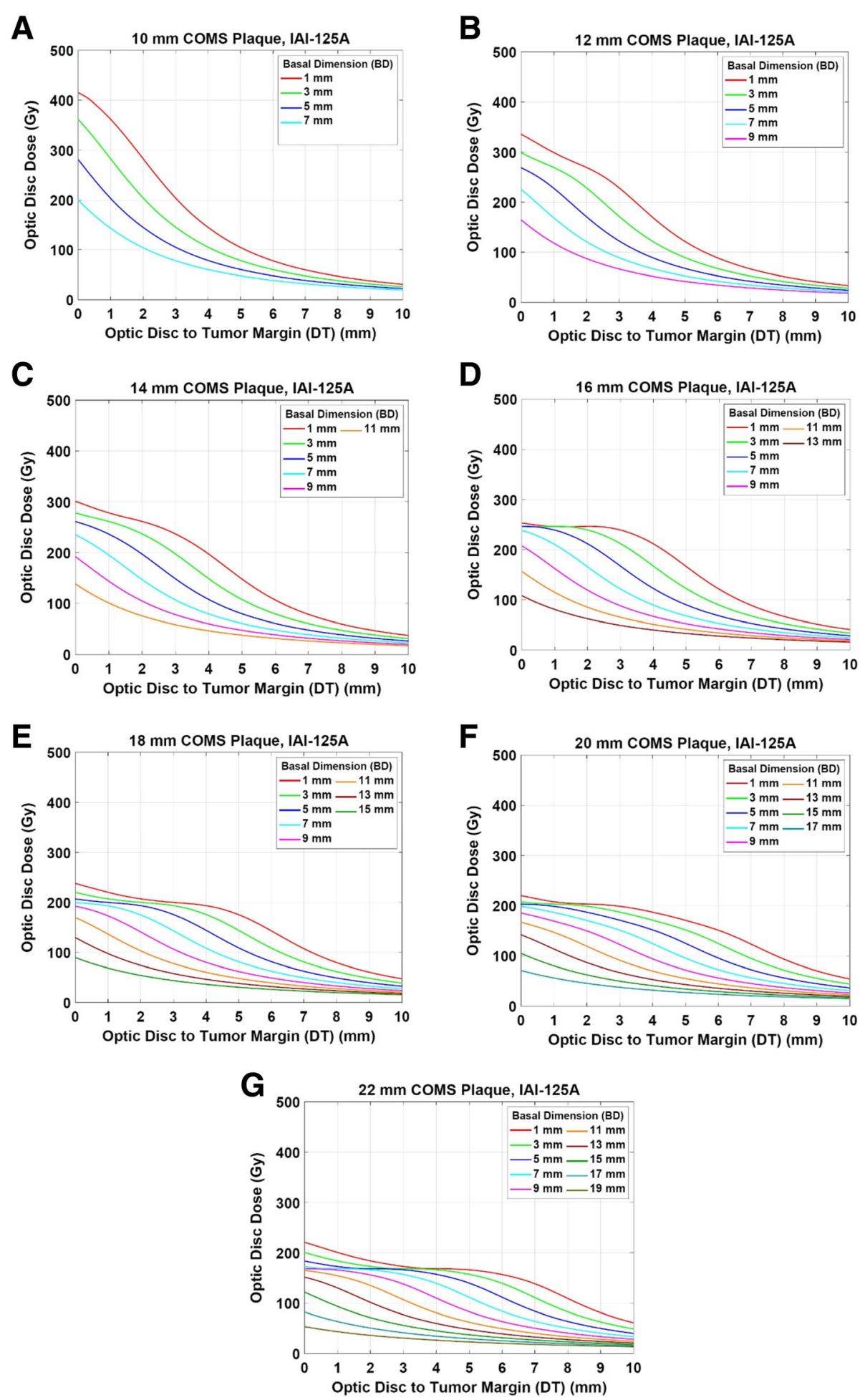

Fig. 2 a-g Optic disc dose as a function of optic disc-to-tumor margin distance (DT) for various tumor basal dimensions (BD) for seven COMS plaques loaded with ${ }^{125}$ seeds (model IAl-125A) when 85 Gy is prescribed to a central-axis depth of $5 \mathrm{~mm}$

( 4.4\%) between the two studies at the lacrimal gland point for plaque positions $\# 1$ and \#3 than at the other dose points.
This study showed that optic disc dose and macula dose strongly depend on distance from tumor margin (DT and MT) and tumor basal dimension (BD and BM). 
A

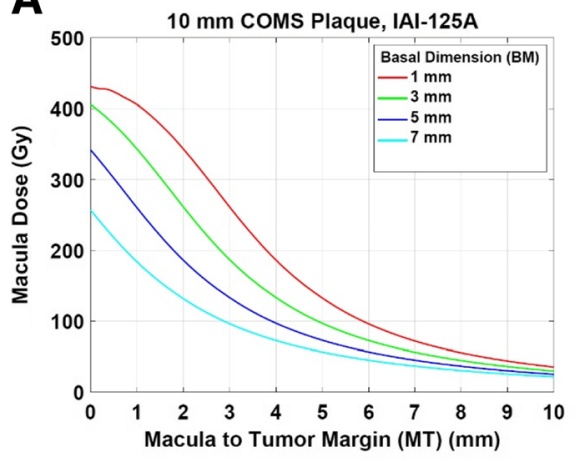

C

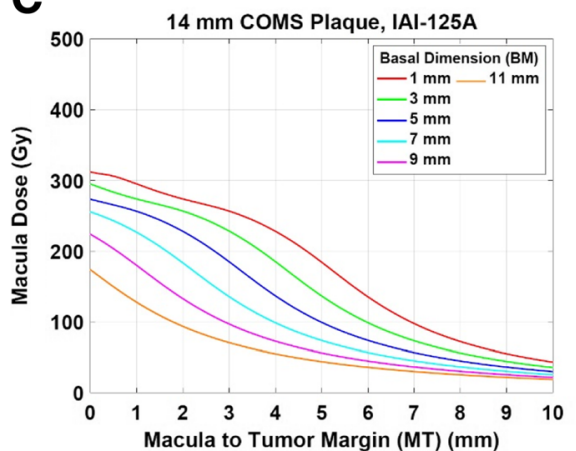

E

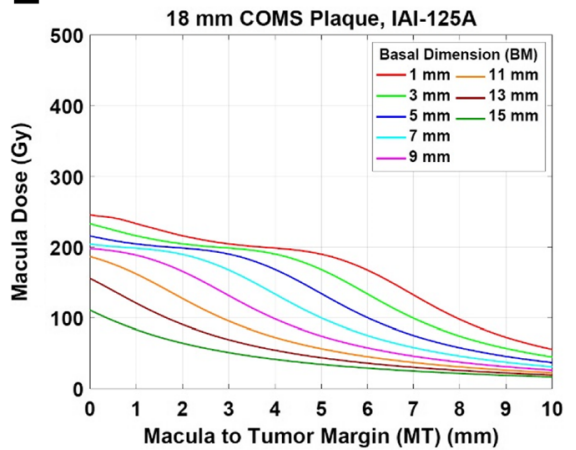

B

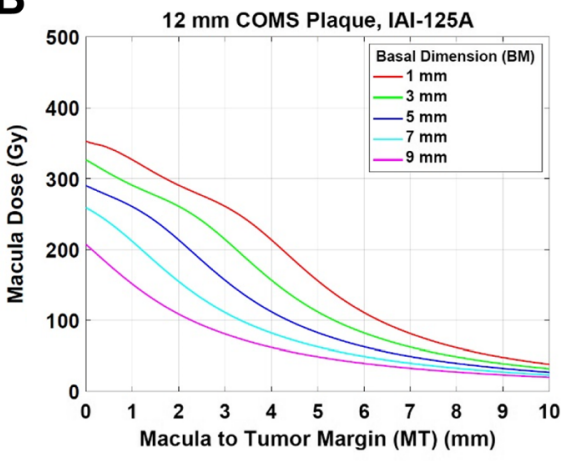

D

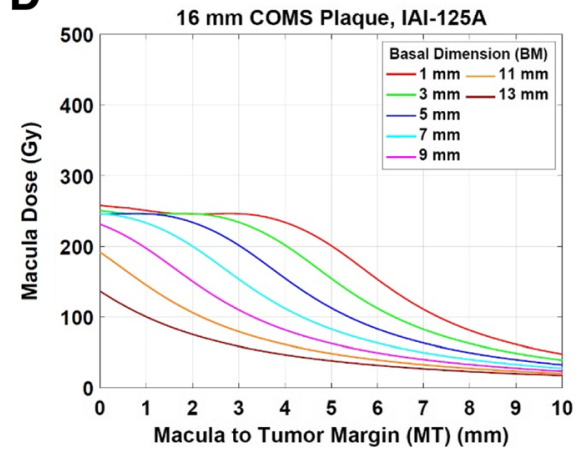

F

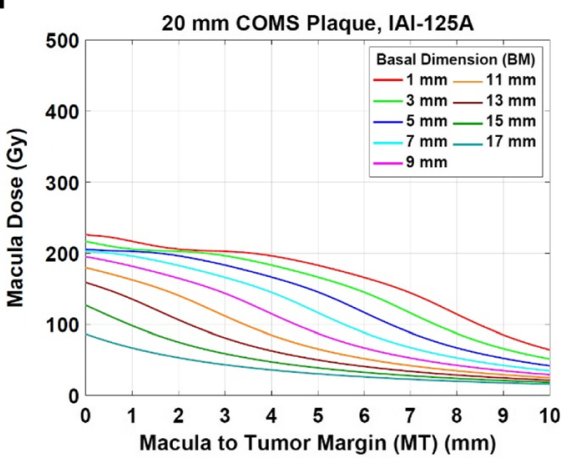

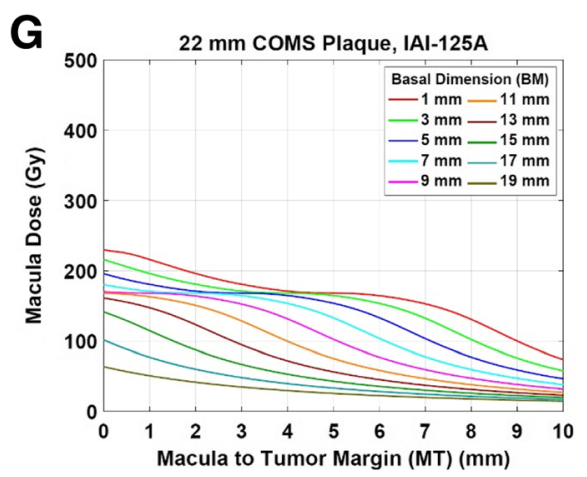

Fig. 3 a-g Macula dose as a function of macula-to-tumor margin distance (MT) for various tumor basal dimensions (BM) for seven COMS plaques loaded with ${ }^{125}$ seeds (model IAl-125A) when 85 Gy is prescribed to a central-axis depth of $5 \mathrm{~mm}$ 
Table 3 Dose conversion factors (ratios of total reference air kerma per seed) for different prescription depths (1 mm $10 \mathrm{~mm}$ in $1 \mathrm{~mm}$ intervals) for standard COMS plaques loaded with ${ }^{125}$ I seeds (model IAI-125A). A reference depth for dose conversion factors is $5 \mathrm{~mm}$

\begin{tabular}{llllllll}
\hline $\begin{array}{l}\text { Prescription } \\
\text { depth }(\mathrm{mm})\end{array}$ & \multicolumn{7}{l}{ Plaque size $(\mathrm{mm})$ in diameter } \\
\cline { 2 - 8 } & 10 & 12 & 14 & 16 & 18 & 20 & 22 \\
\hline 1 & 0.29 & 0.33 & 0.37 & 0.42 & 0.44 & 0.47 & 0.47 \\
2 & 0.41 & 0.45 & 0.48 & 0.52 & 0.55 & 0.58 & 0.59 \\
3 & 0.57 & 0.60 & 0.63 & 0.66 & 0.68 & 0.70 & 0.71 \\
4 & 0.77 & 0.78 & 0.80 & 0.81 & 0.83 & 0.84 & 0.85 \\
5 & 1.00 & 1.00 & 1.00 & 1.00 & 1.00 & 1.00 & 1.00 \\
6 & 1.27 & 1.25 & 1.23 & 1.22 & 1.20 & 1.18 & 1.18 \\
7 & 1.59 & 1.54 & 1.50 & 1.47 & 1.43 & 1.40 & 1.38 \\
8 & 1.95 & 1.88 & 1.82 & 1.76 & 1.69 & 1.64 & 1.61 \\
9 & 2.37 & 2.27 & 2.18 & 2.10 & 2.00 & 1.92 & 1.87 \\
10 & 2.84 & 2.71 & 2.59 & 2.48 & 2.34 & 2.24 & 2.17 \\
\hline
\end{tabular}

In the COMS protocols, coordinates for the optic disc and macula are determined by the combination of these two parameters. Hence, the two parameters determine optic disc dose and macula dose. At close proximity (up to about $1 \mathrm{~cm}$ ) of ${ }^{125}$ I seeds (plaque), the inverse square law effect is severe. At farther distances, however, the radial dose function for ${ }^{125}$ I seeds drastically decreases with distance because of its low photon energy (average energy: $28 \mathrm{keV}$ ) and consequently, rapid dose fall-off is observed. As a result, optic disc dose and macula dose decrease as DT and MT increase (Figs. 2 and 3), respectively. Optic disc dose and macula dose also decrease as BD and BM increase (Figs. 2 and 3), respectively because optic disc and macula become far away from the center of tumor as BD and BM increase, respectively. For plaques $\geq 16 \mathrm{~mm}$, however, there are regions in which dose does not change much with distance (Figs. 2d-g and $3 \mathrm{~d}-\mathrm{g})$. This occurs particularly for small basal dimensions at a tumor margin-to-critical structure distance $\leq 5 \mathrm{~mm}$. As shown in Fig. 4a, for BD of $5 \mathrm{~mm}$, as DT increases, an optic dose point becomes far away from seed $\# 21$ but simultaneously close to seeds \#12 and \#5. As a result, optic disc dose points between $2 \mathrm{~mm}$ and $4 \mathrm{~mm}$ of DT are in the same dose color map (three yellow dots in Fig. 4a) and optic disc dose is invariant within that region. On the other hand, for $\mathrm{BD}$ of $13 \mathrm{~mm}$, an optic disc dose point becomes far away from seeds $\# 12$ and \#5 with increasing DT. Therefore, each dose point between $2 \mathrm{~mm}$ and $4 \mathrm{~mm}$ is located in a different dose color map and optic disc dose decreases as DT increases (Fig. 4b). For the same reason, similar patterns are observed in macula dose (Fig. 3).

Optic disc dose and macula dose also have dependence on prescription depth and plaque size. The dependence can be explained with trends of dose conversion factors (Table 3) and TRAK values per seed (Table 4) as follows. First, for each plaque size, TRAK per seed to obtain a prescribed dose to a prescription depth increases with increasing prescription depth because a deeper prescription depth requires higher TRAK per seed. Hence, dose conversion factors increase with prescription depth. Second, for each prescription depth, TRAK per seed does not continuously decrease with increasing plaque size due to the number of seeds used in each COMS plaque (e.g., 24 seeds for $20 \mathrm{~mm}$ plaque and 21 seeds for $22 \mathrm{~mm}$ plaque). Thus, dose conversion factors do not always continuously increase (depth $<5 \mathrm{~mm}$ ) or decrease (depth $>5 \mathrm{~mm}$ ) with increasing plaque size. Third, TRAK per seed increases more rapidly with increasing prescription depth for smaller plaques than for larger plaques. The following example for seed model IAI-125A supports this trend. For the $10 \mathrm{~mm}$ plaque, TRAK per seed increases from $299.9 \mu \mathrm{Gym}^{2}$ to $2923.5 \mu \mathrm{Gym}^{2}$ (9.7-fold increase) when a prescription depth increases from $1 \mathrm{~mm}$ to $10 \mathrm{~mm}$. On the other hand, for the $22 \mathrm{~mm}$ plaque, the increase of TRAK per seed by the depth increase from $1 \mathrm{~mm}$ to $10 \mathrm{~mm}$ is 4.6 -fold (from 157.3 $\mu \mathrm{Gym}^{2}$ to $718.6 \mu \mathrm{Gym}^{2}$ ). Thus, dose conversion factors increase more rapidly with increasing prescription depth for smaller plaques than for larger plaques, resulting in the increase in dose conversion factors with plaque size at a depth $<5 \mathrm{~mm}$ but the decrease with plaque size at a depth $>5 \mathrm{~mm}$.

There exist dose differences among seed models. The differences are caused by differences of the parameters used in the AAPM TG-43 U1 dosimetry formalism which result from different seed geometry and internal construction among three seed models [16, 19]. As reported in the Results, the differences can be fairly significant (up to $7.7 \%$ ) and similar results were reported by Thomson et al. [19]. Thomson et al. performed MC calculations for a $16 \mathrm{~mm}$ COMS plaque loaded with ${ }^{125} \mathrm{I}$ seeds under TG-43 assumptions (i.e., a homogeneous medium) and showed that doses differed by up to $11 \%$ for different seed models [19].

The results presented in this study will be beneficial to the clinic using ${ }^{125}$ I COMS plaques and help improve current clinical practice as follows. First, this study would allow clinicians to estimate optic disc dose and macula dose without dose calculations. Figures 2 and 3 and Table 3 or the data in additional files can be easily looked up as in the example discussed in the Results. In this study, the calculations were based on a prescribed dose of $85 \mathrm{~Gy}$. In clinical cases where a prescribed dose is different from 85 Gy (e.g., re-irradiation or treatment of benign lesions), a dose scaling factor (prescribed dose (Gy)/85 Gy) can be multiplied by optic disc dose or macula dose obtained in this study to estimate dose to the 


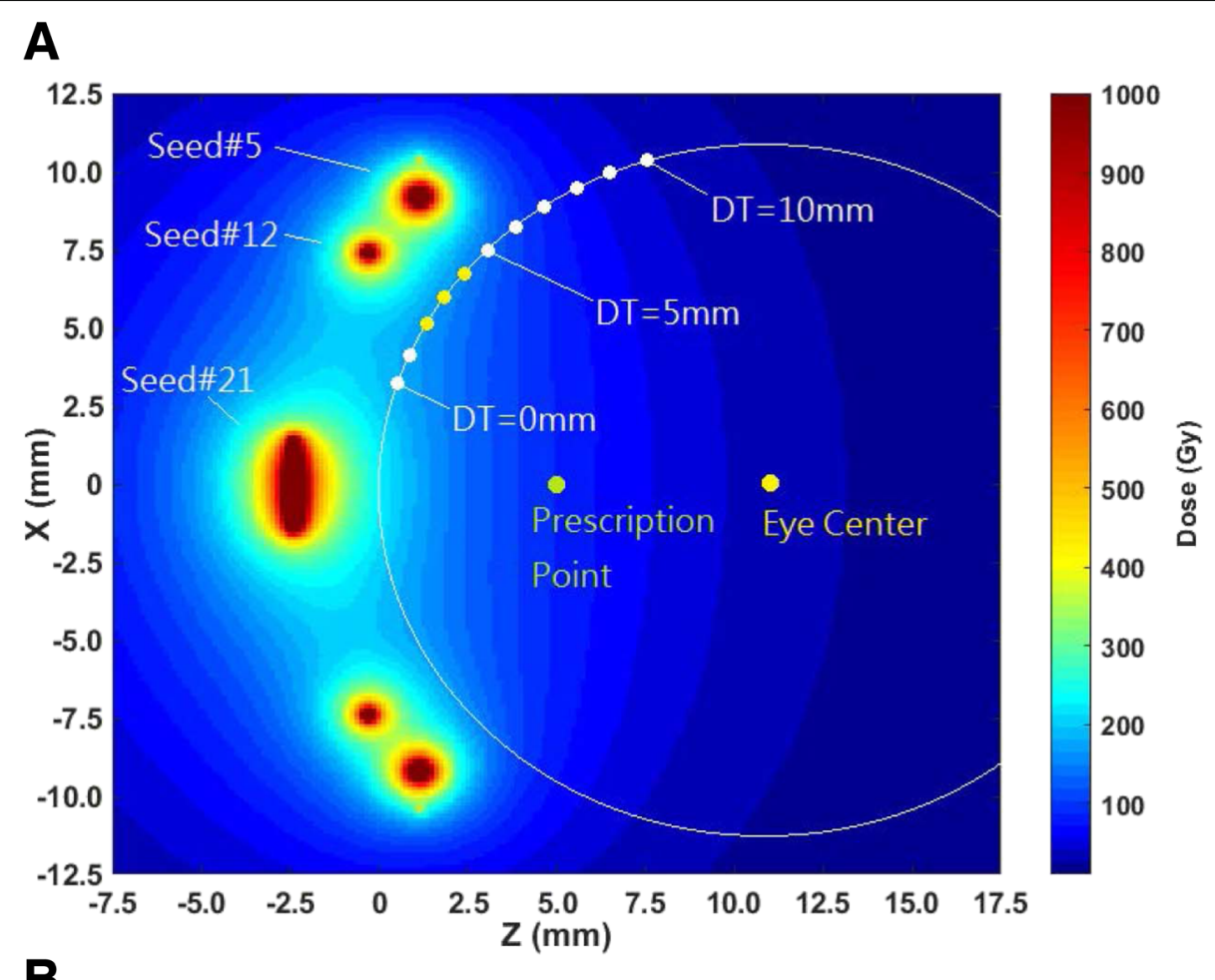

B

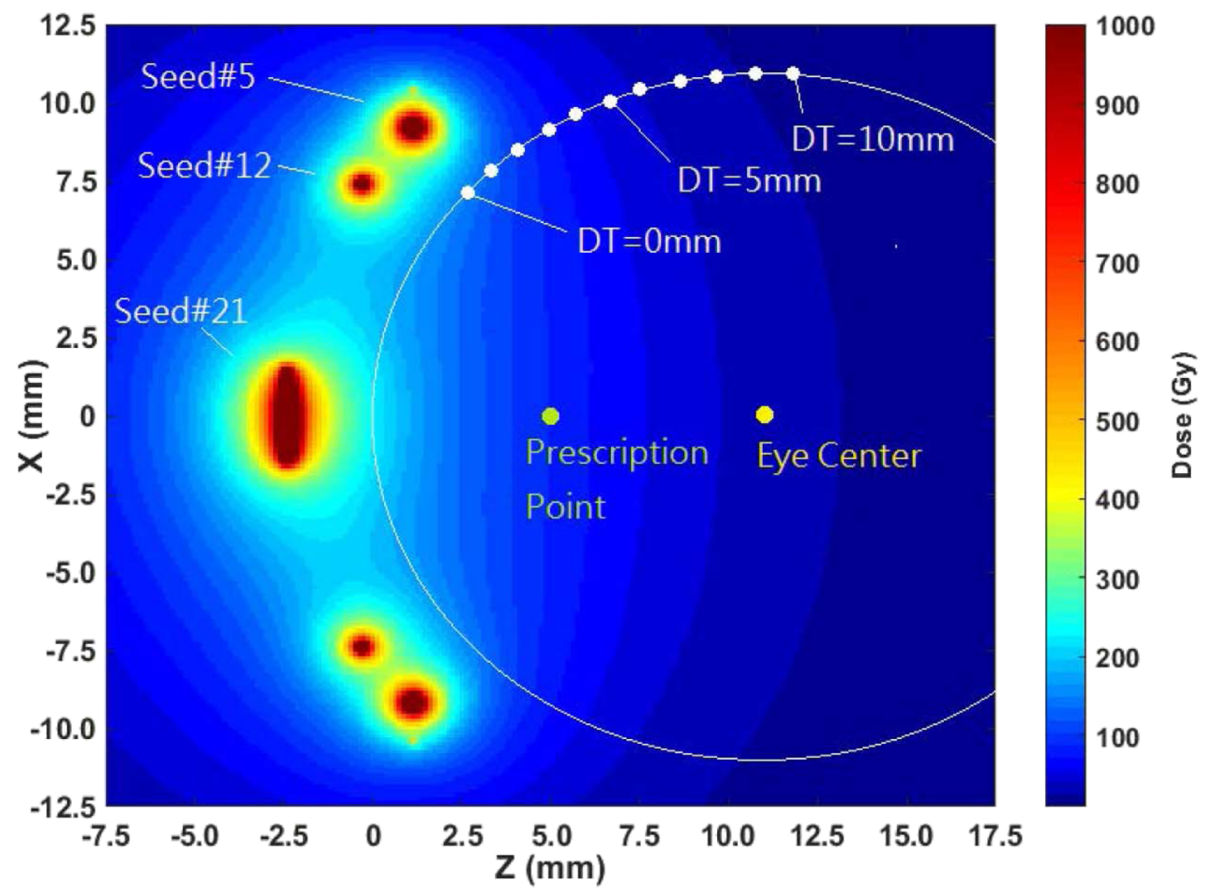

Fig. 4 Optic disc dose clouds for a $22 \mathrm{~mm}$ COMS plaque loaded with ${ }^{125}$ (model: IAl-125A) seeds for a tumor with a 5 mm basal dimension (BD) and $\mathbf{b} 13 \mathrm{~mm}$ basal dimension (BD). The positions in yellow (DT = $2 \mathrm{~mm}-4 \mathrm{~mm}$ ) in the a represent where dose does not change much

critical structures. Second, if an estimated dose to the OARs is high enough to be paid attention to, clinicians may take an action to reduce dose to the OARs by prescribing to a different depth or by the use of a notched plaque [20]. As discussed in the example in the Results, prescribing 85 Gy to $4 \mathrm{~mm}$ (recent ABS guidelines [18]: prescribing at the tumor apex for all medium-sized choroidal melanomas) can give lower dose to the optic disc 
Table 4 TRAK per seed (in $\mu \mathrm{Gym}^{2}$ ) for different prescription depths (1 mm - $10 \mathrm{~mm}$ in $1 \mathrm{~mm}$ intervals) for standard COMS plaques loaded with ${ }^{125}$ I seeds (model IAI-125A)

\begin{tabular}{llllllll}
\hline $\begin{array}{l}\text { Prescription } \\
\text { depth }(\mathrm{mm})\end{array}$ & \multicolumn{7}{l}{ Plaque size $(\mathrm{mm})$ in diameter } \\
\cline { 2 - 9 } & 10 & 12 & 14 & 16 & 18 & 20 & 22 \\
\hline 1 & 299.9 & 226.3 & 161.2 & 191.6 & 134.8 & 132.3 & 157.3 \\
2 & 424.9 & 306.2 & 212.2 & 241.0 & 168.0 & 162.0 & 194.2 \\
3 & 587.2 & 406.6 & 274.4 & 301.3 & 206.3 & 195.8 & 234.6 \\
4 & 788.9 & 530.2 & 349.5 & 373.9 & 251.2 & 234.7 & 280.1 \\
5 & 1030.6 & 677.1 & 438.1 & 459.8 & 303.2 & 279.4 & 331.2 \\
6 & 1311.2 & 848.0 & 540.9 & 559.7 & 363.2 & 330.7 & 389.3 \\
7 & 1634.7 & 1045.2 & 659.3 & 675.4 & 432.6 & 389.9 & 455.7 \\
8 & 2008.7 & 1274.1 & 797.3 & 810.4 & 513.4 & 458.6 & 532.3 \\
9 & 2438.7 & 1536.9 & 955.4 & 965.1 & 605.8 & 537.1 & 619.8 \\
10 & 2923.5 & 1833.6 & 1133.9 & 1140.1 & 710.4 & 626.0 & 718.6 \\
\hline
\end{tabular}

than prescribing 85 Gy to $5 \mathrm{~mm}$ (COMS protocols [14]: prescribing at $5 \mathrm{~mm}$ for the tumor apex $<5 \mathrm{~mm}$ and to the apex for the tumor apex $\geq 5 \mathrm{~mm}$ [15]) (112 Gy vs. 145 Gy) due to lower TRAK per seed at a shallower depth. For the tumor apex $\geq 5 \mathrm{~mm}$, prescribing dose to a shallower depth would reduce optic disc dose but tumor coverage can be compromised. Third, this study would enable clinicians to correlate clinical outcomes for vision with optic disc dose or macula dose. There have been no good published correlation data between clinical outcomes and radiation dose. If clinical outcomes for vision are available along with corresponding distance from tumor margin (DT and MT), tumor basal dimensions (BD and $\mathrm{BM}$ ), plaque size, prescription depth, prescribed dose and seed model, one can correlate the outcomes with optic disc dose and macula dose which can be looked up from the results presented in this study. From the correlation data, clinicians can anticipate outcomes for vision for a given clinical situation and find a possible way to reduce dose to the critical structures before treatment. Furthermore, using the correlation data, tolerance dose to the optic disc or macula in ocular brachytherapy, which has not been known yet, can be investigated.

\section{Conclusions}

This study has comprehensively examined optic disc dose and macula dose as a function of distance from tumor margin in ocular brachytherapy using ${ }^{125} \mathrm{I}$ COMS plaques and has shown that dose to the critical structures has dependence on multiple parameters such as distance from tumor margin, tumor basal dimension, prescription depth, plaque size, and seed model. In any clinic or institution utilizing ${ }^{125}$ I COMS plaques, the dosimetry data provided in this study can be looked up to estimate optic disc dose and macula dose without dose calculations in a TPS.

\section{Additional files}

Additional file 1: 4 figures: optic disc dose (1 for each seed model, 2301 and 125.S16) and macula dose (1 for each seed model, 2301 and I25.S16). (ZIP $5849 \mathrm{~kb})$

Additional file 2: 2 tables: dose conversion factors for two seed models (2301 and I25.S16). (ZIP $23 \mathrm{~kb}$ )

\section{Abbreviations \\ AAPM: American Association of Physicists in Medicine: ABS: American Brachytherapy Society; BD: Tumor basal dimension at center in the direction from optic disc; BM: Tumor basal dimension at center in the direction from macula; COMS: Collaborative Ocular Melanoma Study; DT: Distance from optic disc to tumor margin; MT: Distance from macula to tumor margin; OARs: Organs at risk; $S_{k}$ : Air-kerma strength; TG: Task group; TPS: Treatment planning system; TRAK: Total reference air kerma}

\section{Acknowledgements \\ None}

Funding

None.

Availability of data and materials

Not applicable.

\section{Authors' contributions}

YCL analyzed data and wrote a manuscript. SCL performed calculations and collected data. YK designed the study and analyzed data. All authors read and approved the final manuscript.

Ethics approval and consent to participate

Not applicable.

Consent for publication

Not applicable.

\section{Competing interests}

The authors declare that they have no competing interests.

\section{Publisher's Note}

Springer Nature remains neutral with regard to jurisdictional claims in published maps and institutional affiliations.

Received: 22 August 2018 Accepted: 30 October 2018

Published online: 13 November 2018

\section{References}

1. Fabian ID, Tomkins-Netzer O, Stoker I, Arora AK, Sagoo MS, Cohen VM Secondary enucleations for uveal melanoma: a 7-year retrospective analysis. Am J Ophthalmol. 2015;160(6):1104-10 e1.

2. Nag S, Quivey JM, Earle JD, Followill D, Fontanesi J, Finger PT, et al. The American brachytherapy society recommendations for brachytherapy of uveal melanomas. Int J Radiat Oncol Biol Phys. 2003;56(2):544-55.

3. Chiu-Tsao ST, Astrahan MA, Finger PT, Followill DS, Meigooni AS, Melhus CS, et al. Dosimetry of (125)I and (103)Pd COMS eye plaques for intraocular tumors: report of task group 129 by the AAPM and ABS. Med Phys. 2012; 39(10):6161-84

4. Tseng V, Coleman A, Zhang Z, McCannel T. Complications from plaque versus proton beam therapy for choroidal melanoma: a qualitative systematic review. J Cancer Ther. 2016;7(3):169-85.

5. Sagoo MS, Shields CL, Mashayekhi A, Freire J, Emrich J, Reiff J, et al. Plaque radiotherapy for juxtapapillary choroidal melanoma overhanging the optic disc in 141 consecutive patients. Arch Ophthalmol. 2008;126(11):1515-22.

6. Finger PT. Finger's "slotted" eye plaque for radiation therapy: treatment of juxtapapillary and circumpapillary intraocular tumours. Br J Ophthalmol. 2007;91(7):891-4. 
7. Berry JL, Dandapani SV, Stevanovic M, Lee TC, Astrahan M, Murphree AL, et al. Outcomes of choroidal melanomas treated with eye physics: a 20-year review. JAMA Ophthalmol. 2013;131(11):1435-42.

8. Karlsson M, Nilsson J, Lundell M, Carlsson TA. Monte Carlo dosimetry of the eye plaque design used at the St. Erik eye hospital for (125)l brachytherapy. Brachytherapy. 2014;13(6):651-6.

9. Shields CL, Shields JA, Cater J, Gunduz K, Miyamoto C, Micaily B, et al. Plaque radiotherapy for uveal melanoma: long-term visual outcome in 1106 consecutive patients. Arch Ophthalmol. 2000;118(9):1219-28.

10. Melia BM, Abramson DH, Albert DM, Boldt HC, Earle JD, Hanson WF, et al. Collaborative ocular melanoma study (COMS) randomized trial of I-125 brachytherapy for medium choroidal melanoma. I. Visual acuity after 3 years COMS report no. 16. Ophthalmology. 2001;108(2):348-66.

11. Shields CL, Naseripour M, Cater J, Shields JA, Demirci H, Youseff A, et al. Plaque radiotherapy for large posterior uveal melanomas $(>$ or $=8-\mathrm{mm}$ thick) in 354 consecutive patients. Ophthalmology. 2002;109(10):1838-49.

12. Finger PT, Chin KJ, Duvall G. Palladium-103 for Choroidal Melanoma Study G. Palladium-103 ophthalmic plaque radiation therapy for choroidal melanoma: 400 treated patients. Ophthalmology. 2009;116(4):790-6 6 e1.

13. Sagoo MS, Shields CL, Emrich J, Mashayekhi A, Komarnicky L, Shields JA. Plaque radiotherapy for juxtapapillary choroidal melanoma: treatment complications and visual outcomes in 650 consecutive cases. JAMA Ophthalmol. 2014;132(6):697-702.

14. COMS Manual of Procedures (Chapter 12. Radiation Therapy). http://pages. jh.edu/wctb/coms/manual/coms_chap12.pdf. Accessed 5 Nov 2018.

15. Rivard MJ, Chiu-Tsao ST, Finger PT, Meigooni AS, Melhus CS, Mourtada F, et al. Comparison of dose calculation methods for brachytherapy of intraocular tumors. Med Phys. 2011;38(1):306-16.

16. Rivard MJ, Coursey BM, DeWerd LA, Hanson WF, Huq MS, Ibbott GS, et al. Update of AAPM task group no. 43 report: a revised AAPM protocol for brachytherapy dose calculations. Med Phys. 2004;31(3):633-74.

17. Rivard MJ, Butler WM, DeWerd LA, Huq MS, Ibbott GS, Meigooni AS, et al. Supplement to the 2004 update of the AAPM task group no. 43 report. Med Phys. 2007;34(6):2187-205.

18. American Brachytherapy Society - Ophthalmic Oncology Task Force. Electronic address pec, Committee AO. The American brachytherapy society consensus quidelines for plaque brachytherapy of uveal melanoma and retinoblastoma. Brachytherapy. 2014;13(1):1-14.

19. Thomson RM, Rogers DW. Monte Carlo dosimetry for $125 \mathrm{l}$ and 103Pd eye plaque brachytherapy with various seed models. Med Phys. 2010;37(1):368-76.

20. Hegde JV, McCannel TA, McCannel CA, Lamb J, Wang P-C, Veruttipong D, et al. Juxtapapillary and circumpapillary choroidal melanoma: globe-sparing treatment outcomes with iodine-125 notched plaque brachytherapy. Graefes Arch Clin Exp Ophthalmol. 2017:255(9):1843-50.

Ready to submit your research? Choose BMC and benefit from:

- fast, convenient online submission

- thorough peer review by experienced researchers in your field

- rapid publication on acceptance

- support for research data, including large and complex data types

- gold Open Access which fosters wider collaboration and increased citations

- maximum visibility for your research: over $100 \mathrm{M}$ website views per year

At $\mathrm{BMC}$, research is always in progress.

Learn more biomedcentral.com/submissions 